\title{
Análise do Desempenho do Grupo de Garantia da Qualidade em Projetos de Software
}

\author{
Felipe Furtado ${ }^{1,2}$, Ana Carla Barros ${ }^{2}$, Valéria Moura ${ }^{2}$, Luciana Valadares ${ }^{2}$, \\ Mariana Xavier ${ }^{2}$ \\ ${ }^{1}$ Centro de Informática da Universidade Federal de Pernambuco (UFPE) \\ Caixa Postal 7851, Cidade Universitária - 50.732-970 - Recife - PE - Brasil \\ ${ }^{2}$ C.E.S.A.R - Centro de Estudos e Sistemas Avançados do Recife \\ Rua Bione, $n^{\circ}$ 220, Cais do Apolo 50.0303-90, Recife - PE, Brasil \\ ffelipe.furtado, ana.carla, valeria.moura, luciana.valadares, \\ mariana.xavier\} de cesar.org.br
}

\begin{abstract}
Software development organizations are become more and more concerned about the process used to develop products with high quality. One of the challenges is to measure if the quality team's investment to define and audit process is reflected in the project's results. This paper describes the experience of a CMMI level 3 organization to analyze the impact of software quality assurance activities and its correlation with the project performance.
\end{abstract}

Resumo. Cada vez mais as organizações de desenvolvimento de software estão preocupadas com o processo utilizado para desenvolver produtos com alta qualidade. Um dos grandes desafios é mensurar se o investimento realizado para manter uma equipe responsável pelas atividades de definição e auditoria de processo é refletido nos resultados dos projetos. Este artigo relata a experiência de uma organização avaliada no nível 3 do CMMI na análise de impacto das atividades de $S Q A^{l}$ e sua correlação com os indicadores de sucesso do projeto.

\section{Introdução}

O C.E.S.A.R, Centro de Estudos e Sistemas Avançados do Recife, é um instituto privado de pesquisa, desenvolvimento e inovação criado em 1996. Desde sua criação o Grupo de Garantia da Qualidade do C.E.S.A.R vem trabalhando na definição e melhoria do processo de desenvolvimento de software da organização. Hoje, a empresa possui um processo maduro aderente ao nível 3 do CMMI [SEI 2006] com um programa de melhoria contínua de processo [Chrissis et all 2007].

Segundo Gibson [Gibson et al 2006], a melhoria de processos baseada no CMMI tem sido acompanhada por excelentes resultados quantitativos nos custos, cronograma, produtividade, retorno de investimento (ROI), satisfação do cliente e qualidade do produto. Entretanto, para avaliarmos a melhoria de cada um desses tópicos na organização é necessário que haja um processo de medição [SEI 2006], [Softex 2005].

\footnotetext{
1 SQA: do inglês, Software Quality Assurance. Refere-se às atividades de garantia da qualidade de software.
} 
Os indicadores são essenciais ao planejamento e controle dos processos das organizações [Takashina et al 1996]. Dessa forma, como parte do programa de melhoria contínua da organização, foi iniciado um estudo de indicadores que avaliassem o impacto do uso do processo de desenvolvimento de software nos resultados dos projetos. As seguintes questões foram levantadas:

i. Quais os benefícios práticos que o processo traz ao projeto em termos de melhoria da qualidade dos produtos gerados?

ii. O uso correto do processo implica em gerarmos um produto com qualidade?

iii. Qual a importância de adaptar, apoiar e verificar o uso do processo no projeto?

Para tentar responder esses questionamentos, foi avaliada a correlação entre as atividades de SQA e algumas dimensões da qualidade do produto entregue. Com base nessa correlação foi analisado o desempenho do grupo de garantia da qualidade em projetos de software.

Este relato está organizado da seguinte maneira: inicialmente são apresentados os indicadores das atividades de garantia de qualidade utilizados pela organização. Em seguida é apresentado o processo para estudo da correlação e realizada uma análise dos resultados obtidos. Por fim, são apresentadas as conclusões e trabalhos futuros.

\section{Indicadores das Atividades de Garantia da Qualidade}

Ao definir o processo de Garantia da Qualidade aderente ao CMMI3, alguns indicadores foram especificados para esta área [Paulk et al 1999]. Atualmente existem quatro indicadores utilizados para avaliar as atividades operacionais do grupo de Garantia da Qualidade. Todos eles são analisados mensalmente em relação a cada projeto individual e de forma consolidada de toda a área de qualidade.

i. Variação do esforço de SQA nos projetos: indica a variação entre o esforço planejado e o esforço realizado do engenheiro de qualidade nos projetos.

ii. Realização das auditorias planejadas nos projetos: indica a razão entre as auditorias de processo realizadas e planejadas.

iii. Aderência dos projetos nas auditorias de processo: indica o quanto os projetos estão aderentes ao processo definido para o projeto, conforme mostra a figura 1. Esta aderência pode representar mais de um subprocesso por mês, por exemplo, gerenciamento de requisitos e planejamento de projeto.

iv. Taxa de resolução dos problemas encontrados em auditoria de processo: mostra a capacidade dos projetos em resolver os desvios de processo identificados pelo engenheiro de qualidade. 


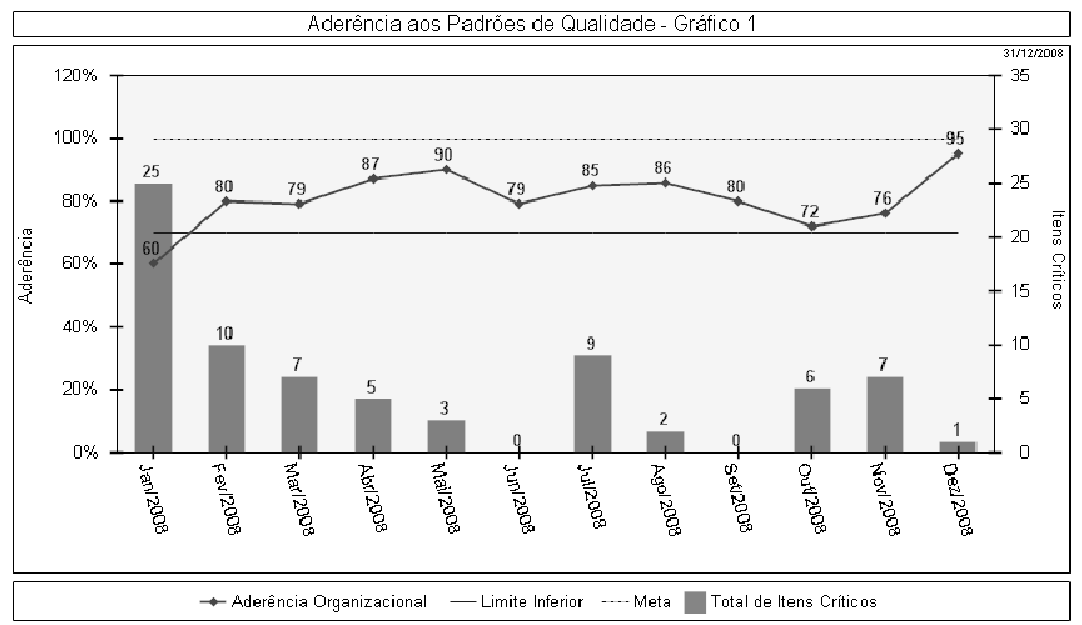

Figura 1. Gráfico do indicador de aderência de processo dos projetos ${ }^{2}$

Esse conjunto de indicadores se mostrou útil ao longo do tempo em termos de acompanhamento da atuação do grupo de Garantia da Qualidade nos projetos. Porém, eles não eram suficientes como forma de quantificar se essas atividades de qualidade estavam relacionadas ao sucesso dos projetos. Surgiu então na organização a necessidade de realizar um estudo para analisar se existe uma correlação entre as atividades do grupo de garantia da qualidade e o resultado do projeto.

\section{Processo para Análise do Desempenho}

Diante desta necessidade, um processo de análise foi definido, como mostra a figura 2. As subseções seguintes detalham as atividades realizadas durante este processo.

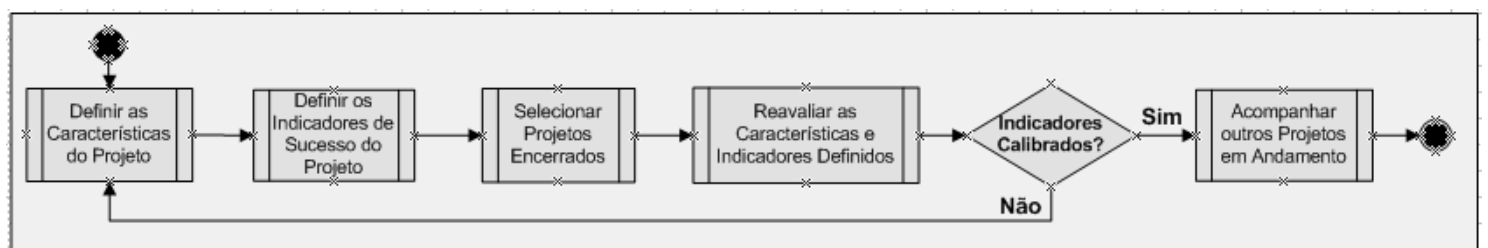

Figura 2. Processo para análise do desempenho

\subsection{Definir as Características do Projeto}

O primeiro passo foi definir quais as principais características de um projeto que podem ser relacionadas com as atividades de garantia da qualidade. Segue abaixo uma breve explicação de cada uma delas com a respectiva forma de classificação:

Tabela 1. Características do projeto

\begin{tabular}{|l|l|}
\hline Característica & Descrição e Classificação \\
\hline $\begin{array}{l}\text { Aderência em } \\
\text { relação ao uso do } \\
\text { processo definido } \\
\text { para o projeto. }\end{array}$ & $\begin{array}{l}\text { Mede o percentual de aderência do projeto em relação ao uso do } \\
\text { processo definido. Este processo é uma adaptação do processo } \\
\text { organizacional de desenvolvimento de software. }\end{array}$ \\
\hline
\end{tabular}

\footnotetext{
${ }^{2}$ Por questões confidenciais, esses valores são fictícios e apenas ilustram como o indicador é apresentado.
} 


\begin{tabular}{|l|l|}
\hline & $\begin{array}{l}\text { Sem aderência: até 30\%; Baixa: entre 30\% e 60\%; Média: entre 60\% e } \\
80 \% \text {; Alta: maior ou igual a 80\%. }\end{array}$ \\
\hline $\begin{array}{l}\text { Percentual da } \\
\text { alocação real do } \\
S Q E^{3} \text { no projeto }\end{array}$ & $\begin{array}{l}\text { Mede o percentual real de esforço do SQE no projeto avaliado. Na maior } \\
\text { parte dos casos, o SQE é alocado em vários projetos ao mesmo tempo, } \\
\text { com percentuais de alocação pré-definidos. } \\
\text { Baixo: até 30\%; Médio: entre 30\% e 50\%; Alto: maior ou igual a 50\%. }\end{array}$ \\
\hline $\begin{array}{l}\text { Número de } \\
\text { projetos em que o o } \\
\text { SQE está } \\
\text { alocado }\end{array}$ & $\begin{array}{l}\text { Mede a quantidade de projetos em que o SQE está alocado ao mesmo } \\
\text { tempo. } \\
\text { Baixo: um projeto; Médio: } 2 \text { ou 3 projetos; Alto: maior que 3 projetos. }\end{array}$ \\
\hline $\begin{array}{l}\text { Nível de } \\
\text { colaboração da } \\
\text { equipe do projeto } \\
\text { em relação ao } \\
\text { uso do processo }\end{array}$ & $\begin{array}{l}\text { Mede o quanto a equipe do projeto possui maturidade e/ou resistência em } \\
\text { relação ao uso do processo definido. } \\
\text { Baixo: equipe com baixa maturidade ou alta resistência ao uso do } \\
\text { processo adaptado; Médio: apenas o gerente do projeto e o líder de } \\
\text { equipe são maduros ou não são resistentes ao uso do processo definido; } \\
\text { Alto: toda a equipe é madura ou apresenta baixa resistência no uso do } \\
\text { processo definido. }\end{array}$ \\
\hline $\begin{array}{l}\text { Nível de } \\
\text { colaboração do } \\
\text { cliente em } \\
\text { relação ao uso do } \\
\text { processo }\end{array}$ & $\begin{array}{l}\text { Mede o quanto o cliente colabora com a equipe no uso do processo } \\
\text { definido para o projeto. } \\
\text { Baixo: o cliente não requer nenhum tipo de aderência a um processo } \\
\text { definido; Médio: o cliente em alguns momentos demonstra interesse que o } \\
\text { projeto seja aderente a um processo definido; Alto: o cliente requer total } \\
\text { aderência a um processo definido. }\end{array}$ \\
\hline
\end{tabular}

\subsection{Definir os Indicadores de Sucesso do Projeto}

O segundo passo do processo foi identificar os indicadores que definem o sucesso de um projeto, por exemplo, satisfação do cliente e entregas no prazo, conforme mostra a tabela 2.

Tabela 2. Indicadores de sucesso do projeto

\begin{tabular}{|l|l|}
\hline Indicador & Descrição e Classificação \\
\hline $\begin{array}{l}\text { Satisfação } \\
\text { do cliente }\end{array}$ & $\begin{array}{l}\text { Mede o nível de satisfação do cliente em relação ao projeto. Várias questões } \\
\text { são analisadas, por exemplo, qualidade do produto entregue, prazo de entrega, } \\
\text { visibilidade do acompanhamento do projeto, interação com a equipe do projeto, } \\
\text { dentre outros. Ao final da pesquisa de satisfação, uma nota é dada ao projeto. } \\
\text { Baixa: nota até 3,5 pontos; Média: nota entre 3,5 e 4 pontos; Alta: nota maior ou } \\
\text { igual a 4 e menor ou igual a 5 pontos. }\end{array}$ \\
\hline $\begin{array}{l}\text { Entregas } \\
\text { no prazo }\end{array}$ & $\begin{array}{l}\text { Mede o percentual de entregas ao cliente realizadas no prazo planejado. } \\
\text { Baixa: todas as entregas foram realizadas fora do prazo planejado; Média: } \\
\text { algumas entregas foram realizadas fora do prazo planejado; Alta: todas as } \\
\text { entregas foram realizadas dentro do prazo planejado. }\end{array}$ \\
\hline $\begin{array}{l}\text { Número de de } \\
\text { defeitos } \\
\text { pós- }\end{array}$ & $\begin{array}{l}\text { Mede a quantidade e severidade de defeitos detectados pelo cliente após a } \\
\text { entrega formal de um produto. } \\
\text { Baixo: apenas defeitos de baixa severidade foram encontrados pós-entrega; }\end{array}$ \\
\hline
\end{tabular}

\footnotetext{
${ }^{3} S Q E$ : do inglês, Software Quality Engineer. Refere-se ao Engenheiro de Qualidade de Software que atua no projeto com foco em definição, suporte e auditoria de processo e produto.
} 


\begin{tabular}{|l|l|}
\hline entrega & $\begin{array}{l}\text { Médio: pelo menos um defeito de média severidade foi encontrado pós-entrega; } \\
\text { Alto: pelo menos um defeito de alta severidade foi encontrado pós-entrega. }\end{array}$ \\
\hline Orçamento & $\begin{array}{l}\text { Mede a variação do orçamento realizado ao longo do projeto em relação ao } \\
\text { orçamento planejado no início do projeto. } \\
\text { Baixo: projeto executado com mais de } 5 \% \text { de variação do orçamento planejado; } \\
\text { Médio: projeto executado com até 5\% de variação do orçamento planejado; Alto: } \\
\text { projeto executado dentro do orçamento planejado. }\end{array}$ \\
\hline $\begin{array}{l}\text { Satisfação } \\
\text { da equipe }\end{array}$ & $\begin{array}{l}\text { Mede o nível de satisfação da equipe em relação ao projeto. Várias questões } \\
\text { são analisadas, por exemplo, qualidade de vida, planejamento das atividades } \\
\text { que precisam ser executadas, envolvimento da equipe no acompanhamento dos } \\
\text { riscos do projeto, dentre outros. Ao final da pesquisa de satisfação, uma nota é } \\
\text { dada ao projeto. } \\
\text { Baixa: nota até 3,5 pontos; Média: nota entre 3,5 e } 4 \text { pontos; Alta: nota maior ou } \\
\text { igual a 4 e menor ou igual a 5 pontos. }\end{array}$ \\
\hline
\end{tabular}

\subsection{Análise, Calibração e Acompanhamento dos Indicadores}

Os três últimos passos foram definidos com o objetivo de selecionar os projetos já encerrados que seriam analisados inicialmente. Esse passo foi dividido em três etapas: coletar as características e os indicadores descritos nas duas subseções anteriores no grupo de projetos selecionado; analisar se existe uma correlação entre eles; e identificar se outros fatores externos ao processo também contribuíram para a correlação analisada.

Em seguida foi feita uma reavaliação das características e indicadores previamente definidos, onde alguns deles foram calibrados em relação ao mecanismo de coleta e forma de classificação, como definidos nas tabelas 1 e 2 . Finalmente, novos projetos foram acompanhados com base na calibração do processo definido.

\section{Análise dos Resultados Obtidos}

Com base no processo descrito na seção 3, foi selecionada uma amostra inicial com doze projetos de diferentes características e depois da calibração esta amostra aumentou para vinte e um projetos. As subseções seguintes descrevem os critérios utilizados para quantificar as escalas e as conclusões geradas.

\subsection{Análise dos Critérios}

Para iniciar a análise dos critérios relacionados às características do projeto e aos indicadores de sucesso do projeto, foi necessário definir uma escala numérica para cada item que havia sido pontuado através de conceitos ('A'=Alto, 'M'=Médio, 'B'=Baixo).

No caso das características do projeto, foram definidos pesos de acordo com o julgamento da organização com relação aos itens que promovem melhores condições de atuação do SQE no projeto. Em seguida, cada característica teve seu peso associado entre os conceitos da seguinte forma: conceito 'Alto' equivale ao peso total, conceito 'Médio' equivale a 2/3 e o conceito 'Baixo' equivale a 1/3, conforme mostra a tabela 3.

Tabela 3. Pesos para características do projeto

\begin{tabular}{|l|l|l|l|l|}
\hline Características do Projeto & Peso & A & M & B \\
\hline Aderência do projeto em relação ao uso do processo definido & 30 & 30 & 20 & 10 \\
\hline Percentual da alocação real do SQE no projeto & 15 & 15 & 10 & 5 \\
\hline
\end{tabular}




\begin{tabular}{|l|l|l|l|l|}
\hline Número de projetos em que o SQE está alocado & 15 & 5 & 10 & 15 \\
\hline Nível de colaboração da equipe em relação ao uso do processo & 30 & 30 & 20 & 10 \\
\hline Nível de colaboração do cliente em relação ao uso do processo & 10 & 10 & 6,7 & 3,3 \\
\hline
\end{tabular}

Como mostra a tabela abaixo, no caso dos indicadores de sucesso do projeto, os pesos foram definidos de maneira uniforme entre todos os indicadores, visto que a organização os julga com igual importância e há uma forte relação de dependência entre eles.

Tabela 4. Pesos para indicadores de sucesso do projeto

\begin{tabular}{|l|l|l|l|l|}
\hline Indicadores de Sucesso do Projeto & Peso & A & M & B \\
\hline Satisfação do cliente & 20 & 20 & 13,3 & 6,7 \\
\hline Satisfação da equipe & 20 & 20 & 13,3 & 6,7 \\
\hline Entregas no prazo & 20 & 20 & 13,3 & 6,7 \\
\hline Número de defeitos pós-entrega & 20 & 6,7 & 13,3 & 20 \\
\hline Orçamento & 20 & 20 & 13,3 & 6,7 \\
\hline
\end{tabular}

Através de uma média ponderada desses valores, foram obtidos dois resultados para cada projeto, um para as características do projeto e outro para os indicadores de sucesso do projeto, conforme mostram as colunas 'Resultado' na tabela a seguir.

Tabela 5. Amostra dos projetos analisados ${ }^{4}$

\begin{tabular}{|c|c|c|c|c|c|c|c|c|c|c|c|c|}
\hline \multirow[b]{2}{*}{ Projeto } & \multicolumn{6}{|c|}{ Características do Projeto } & \multicolumn{6}{|c|}{ Indicadores de Sucesso do Projeto } \\
\hline & Aderência & Alocação & Qtd Projetos & $\begin{array}{c}\text { Colaboração } \\
\text { Equipe }\end{array}$ & $\begin{array}{c}\text { Colaboração } \\
\text { Cliente }\end{array}$ & Resultado & $\begin{array}{l}\text { Satisfação } \\
\text { do cliente }\end{array}$ & $\begin{array}{l}\text { Entregas } \\
\text { no prazo }\end{array}$ & $\begin{array}{c}\text { Número de } \\
\text { defeitos pós- } \\
\text { entrega }\end{array}$ & Orçamento & $\begin{array}{l}\text { Satisfação } \\
\text { da equipe }\end{array}$ & Resultado \\
\hline Projeto 1 & A & A & $\mathrm{M}$ & A & $\mathrm{M}$ & 91,67 & A & A & B & M & A & 93,33 \\
\hline Projeto 2 & $\mathrm{M}$ & $B$ & $\mathrm{M}$ & A & M & 71,67 & A & A & M & A & A & 93,33 \\
\hline Projeto 3 & $\mathrm{M}$ & A & $\mathrm{M}$ & A & M & 81,67 & A & A & M & M & A & 86,67 \\
\hline Projeto 4 & M & A & M & M & A & 75,00 & M & A & M & A & M & 80,00 \\
\hline Projeto 5 & A & $M$ & A & $M$ & $M$ & 71,67 & $M$ & A & $B$ & $B$ & $M$ & 73,33 \\
\hline Projeto 6 & A & $B$ & A & $M$ & A & 70,00 & $M$ & $M$ & $B$ & A & $M$ & 80,00 \\
\hline $\begin{array}{l}\text { Projeto } 7 \\
\end{array}$ & $M$ & A & A & $M$ & A & 70,00 & $M$ & $M$ & $B$ & A & $M$ & 80,00 \\
\hline Projeto 8 & A & A & A & A & A & 90,00 & A & $M$ & M & $M$ & M & 73,33 \\
\hline Projeto 9 & $B$ & A & $B$ & A & $M$ & 76,67 & $M$ & $M$ & $M$ & $M$ & A & 73,33 \\
\hline Projeto 10 & M & M & A & A & $\mathrm{M}$ & 71,67 & A & M & $B$ & $M$ & A & 86,67 \\
\hline Projeto 11 & A & $\mathrm{M}$ & $M$ & $B$ & $M$ & 66,67 & $B$ & $\mathrm{M}$ & & A & A & 70,00 \\
\hline Projeto 12 & M & $\mathrm{M}$ & A & A & $B$ & 68,33 & $M$ & $\mathrm{M}$ & $M$ & A & $B$ & 66,67 \\
\hline Projeto 13 & A & $\mathrm{M}$ & $\mathrm{M}$ & $\mathrm{M}$ & $B$ & 73,33 & A & $B$ & $B$ & $M$ & A & 80,00 \\
\hline Projeto 14 & A & A & $\mathrm{M}$ & $M$ & $\mathrm{M}$ & 81,67 & $M$ & A & A & $M$ & A & 73,33 \\
\hline Projeto 15 & A & A & $\mathrm{M}$ & $M$ & A & 85,00 & $B$ & A & & A & A & 76,67 \\
\hline Projeto 16 & $\mathrm{M}$ & $B$ & A & A & A & 70,00 & A & $\mathrm{M}$ & $M$ & $M$ & $M$ & 73,33 \\
\hline Projeto 17 & B & A & A & A & A & 70,00 & M & A & M & M & $\mathrm{M}$ & 73,33 \\
\hline Projeto 18 & A & A & A & A & A & 90,00 & A & M & M & A & M & 80,00 \\
\hline Projeto 19 & $\mathrm{M}$ & B & $\mathrm{M}$ & A & $\mathrm{M}$ & 71,67 & A & A & B & A & A & 100,00 \\
\hline Projeto 20 & $B$ & $\mathrm{M}$ & $B$ & A & $B$ & 68,33 & A & A & B & $\mathrm{M}$ & A & 93,33 \\
\hline Projeto 21 & A & A & A & $B$ & $M$ & 66,67 & A & A & A & A & A & 86,67 \\
\hline
\end{tabular}

Em seguida foi definida uma classificação para analisar estes resultados: Desfavorável (até 70\%), Razoável (entre 70\% e 80\%) e Favorável (maior ou igual a $80 \%)$.

\subsection{Análise da Correlação entre as Características e os Indicadores}

Após a consolidação dos dados, observou-se que:

\footnotetext{
${ }^{4}$ Por questões confidenciais da Organização, esses valores são fictícios e apenas ilustram como os dados das características e indicadores foram coletados e a forma de cálculo da coluna 'Resultado'.
} 
- Quase $70 \%$ dos Projetos que obtiveram resultado Favorável possuíam características também com classificação Favorável;

- $50 \%$ dos Projetos que obtiveram resultado Razoável possuíam características com classificação Desfavorável;

- $75 \%$ dos Projetos que obtiveram resultado Razoável ou Favorável possuíam características também com classificação Razoável ou Favorável;

- Quase $90 \%$ dos Projetos que obtiveram resultado Desfavorável possuíam características também com classificação Desfavorável.

Os dados numéricos referentes às colunas 'Resultado' de cada projeto foram inseridos em uma ferramenta estatística [Minitab 2008] para verificar se havia correlação entre as características levantadas e os resultados do projeto. Para isso foi usado o diagrama de dispersão abaixo. O resultado da correlação foi de 0.731 . Este coeficiente ${ }^{5}$ indica que há uma correlação linear significativa entre as variáveis.

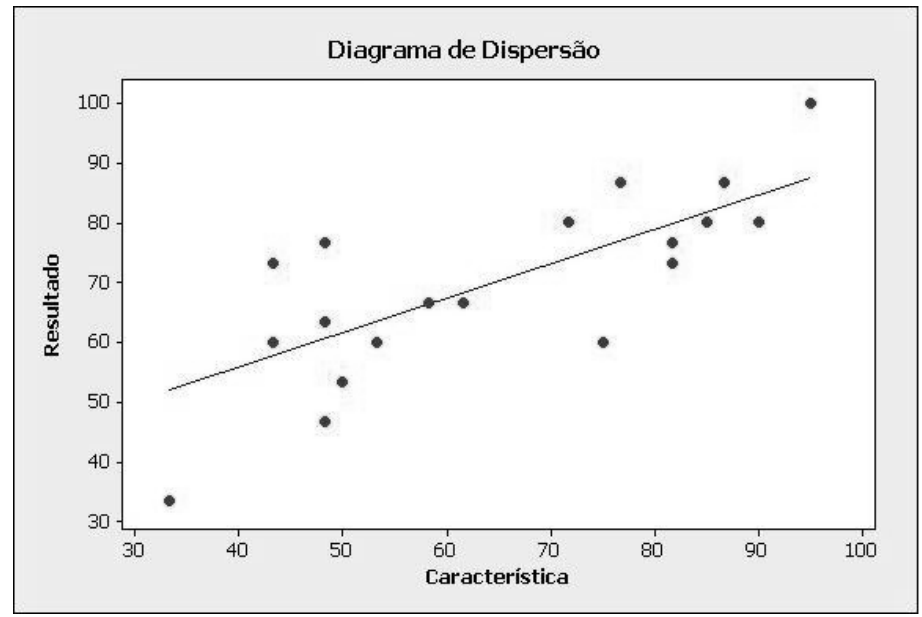

Figura 3. Diagrama de Dispersão

\section{Conclusões e Trabalhos Futuros}

Algumas observações podem ser destacadas partindo da análise dos itens citados anteriormente ao longo do processo de avaliação do indicador de desempenho:

- Foram analisados 21 projetos de diferentes tipos: testes, desenvolvimento, manutenção, design e consultoria;

- Outros fatores externos ao processo também podem ter contribuído com o resultado do projeto, por exemplo, a experiência técnica da equipe e a complexidade dos projetos;

\footnotetext{
${ }^{5}$ Este valor corresponde ao coeficiente de correlação linear que é útil para detectar padrões lineares. É uma medida de quão bem a reta de regressão se ajusta aos dados amostrais e indica que quanto mais próximo do valor 1.00, mais forte é a correlação entre as variáveis. Se o valor absoluto deste coeficiente excede o valor correspondente na tabela Pearson, concluí-se que há correlação linear significativa [Montgomery 2001].
} 
- O escopo de auditoria de processo teve foco em subprocessos variados dentro dos projetos;

- A maturidade da equipe em relação ao uso do processo é um conceito subjetivo;

- A forma de medir a satisfação do cliente pode variar por projeto e ser verificada em momentos diferentes;

- Em alguns projetos existe uma parceria com outros fornecedores e, por isso, nem sempre é possível detectar o número de defeitos encontrados pelo cliente e que tenham sido necessariamente inseridos pela equipe em questão.

Desta forma, pode-se observar a tendência que quando os aspectos favoráveis à equipe de qualidade estão presentes, suas atividades possuem uma relação com os resultados positivos do projeto. De forma similar e mais evidente, observa-se a tendência que quando os aspectos desfavoráveis à equipe de qualidade estão presentes, os resultados do projeto, em geral, não são satisfatórios.

Com base nesta conclusão, os trabalhos futuros são:

- Criar critérios mais objetivos para a mensuração do nível de colaboração da equipe e do cliente;

- Aumentar a amostra dos projetos já encerrados que possam contribuir com a conclusão desta pesquisa;

- Identificar outros atributos que possam influenciar no resultado do projeto;

- Identificar fatores externos que também possam ter influenciado nos resultados encontrados.

\section{Referências}

SEI (2006). CMMI for Development, version 1.2, staged representation. http://www.sei.cmu.edu/pub/documents/06.reports/pdf/06tr008.pdf, CMU/SEI-2006TR-008.

Chrissis B., Korad, M. \& Shrum, S. (2007) CMMI Guidelines for Process Integration and Product Improvement, Second Edition, Addisson-Wesley, EUA.

Gibson, Diane L., Dennis R. Goldenson, and Keith Kost. (2006). "Performance Results of CMMI-Based Process Improvement.” In: CMU/SEI-2006-TR-004, ESC-TR-2006004. Pittsburgh, PA: SEI, Carnegie Mellon University (CMU).

SOFTEX (2005), "MPS.BR - Melhoria de Processo de Software: Guia Geral”.

Takashina, T., Flores, M. (1996), Indicadores da Qualidade e do Desempenho, Editora Qualitymark, 1 a edição.

Paulk et al (1999).The Capability Maturity Model: Guidelines for Improving the Software Process, Addison-Wesley.

Montgomery, D., Peck, E., Vinig, G., Introduction to Linear Regression Analysis, Third Edition, 2001.

Minitab 15, "Software Estatístico", http://www.minitabbrasil.com.br/. Dezembro, 2008. 